\title{
MicroRNA and gene networks in human Hodgkin's lymphoma
}

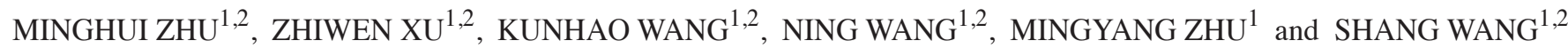 \\ ${ }^{1}$ College of Computer Science and Technology; ${ }^{2}$ Key Laboratory of Symbolic \\ Computation and Knowledge Engineering of Ministry of Education, \\ Jilin University, Changchun, Jilin 130012, P.R. China
}

Received April 4, 2013; Accepted September 24, 2013

DOI: $10.3892 / \mathrm{mmr} .2013 .1741$

\begin{abstract}
There has been significant progress in gene and microRNA (miRNA) research with regard to the morbidity of Hodgkin's lymphoma (HL). However, the regulatory mechanisms of genes and miRNAs have yet to be determined. In the current study, the regulatory association between genes, miRNAs and transcription factors (TFs) was investigated to gain an understanding of the mechanisms and key pathways of HL. The association between TFs and miRNAs, miRNAs and target genes and miRNA and its host gene was examined. To show the regulatory correlation clearly, three regulatory networks were hierarchically constructed: Differentially expressed, associated and global networks. Following comparison and analysis of the similarities and differences among the three networks, a number of key pathways, which showed self-adaptation associations were identified. This included NFאB1 and hsa-miR-9, hsa-miR-196a-1 and its host gene HOXB7, which separately forms a self-adaptation association. The differentially expressed network illuminated the pathogenesis of HL. In addition, the associated network further described the regulatory mechanism associated with HL, including prevention, diagnosis, development and therapy. The current study systematically explains the regulatory mechanisms of HL and supplies comprehensive data associated with HL for further studies. With increasing knowledge of the occurrence, mechanism, improvement, metastasis and treatment, an increased understanding of HL may be achieved.
\end{abstract}

Correspondence to: Professor Zhiwen Xu or Dr Shang Wang, College of Computer Science and Technology, Jilin University, 2699 Qianjin Street, Changchun, Jilin 130012, P.R. China

E-mail:xuzw@jlu.edu.cn

E-mail: wangshang@jlu.edu.cn

Abbreviations: miRNA, microRNA; TFs, transcription factors; targets, target genes; HL, Hodgkin's lymphoma; NCBI, National Center for Biotechnology Information; TFBSs, transcription factor binding sites

Key words: Hodgkin's lymphoma, microRNA, transcription factor, network, pathway, host gene

\section{Introduction}

Hodgkin's lymphoma (HL) is a frequently occurring lymphoid cancer, with an annual incidence rate of three to four novel cases per 100,000 individuals in the Western population (1). As $\mathrm{HL}$ is an aggressive malignancy and is difficult to diagnose at advanced stages, identification of reliable diagnostic markers, regulatory factors and molecular targets is required, and an understanding of the mechanism of HL must be developed. Previous studies have indicated that differentially expressed genes and miRNAs determine the development, metastasis and therapy of HL (2). HL-associated factors (genes and miRNA) are also important.

Transcription factors (TFs) and microRNAs (miRNAs) are prominent regulators of gene expression (3). TFs are specific proteins that activate or repress the transcription of genes by binding to cis-regulatory elements located in the upstream regions of genes (4). Alone or combined with other proteins, TFs regulate gene expression at the transcriptional level. miRNAs are small (21-24 nt) noncoding RNA molecules, which affect gene expression at the post-transcriptional level. As miRNAs repress the translation or degradation of mRNA, they are also capable of controlling key cellular processes, including proliferation, differentiation and apoptosis. A previous study indicated that miRNAs are implicated in a number of human diseases, including leukemia, lung cancer and HL (5). Gibcus et al (1) demonstrated that miRNAs are fundamental in HL by investigating the biogenesis and differential expression of miRNAs, as well as the corresponding potential involvement of miRNAs in the pathogenesis, progression and metastasis.

A number of transcription profiling studies of miRNA transfection have been conducted to investigate the effect of miRNAs on transcription levels. These identified that miRNAs exert a widespread impact on the regulation of their target genes (targets) $(6,7)$. To date, numerous databases, including computational predicted methods (8) and experimentally validated databases $(9,10)$ have provided abundant resources to investigate the association between miRNAs and their targets.

Host genes are the genes to which miRNAs locate. Rodriguez et al (11) indicated that miRNAs are transcribed in parallel with their host transcripts and two transcription classes of miRNAs, exonic and intronic, have been identified. Baskerville et al (12) demonstrated a close correlation between intronic miRNA and its host gene. Intronic miRNA and the 
host gene are coordinately expressed in biological progression and act as potential partners to achieve biological function and affect the alteration of pathways (13).

Molecular biologists and medical researchers have identified numerous differentially expressed genes and miRNAs through a large number of studies on HL (14). However, these studies were are largely based on a single element, gene or miRNA, rendering it difficult to analyze the general pathogenesis of HL. In the current study, the underlying networks of miRNAs, targets of miRNAs, TFs and host genes of miRNAs were investigated to identify the key pathways of HL and understand their control mechanisms in HL. Three types of experimentally validated associations were identified, including miRNA targets, TFs regulating miRNAs and miRNA host genes. Differentially expressed elements and HL-associated elements were manually collected from databases and the literature. A number of predicted TFs were obtained by the P-match method and were considered to be HL-associated genes. Three regulatory networks, differentially expressed, associated and global networks, were constructed based on the associations observed according to the degree of correlation between the elements and HL. The global network was constructed with all the experimentally validated associations at the time of writing. Due to the complexity of constructing all pathways associated with HL, the regulatory associations of differently expressed elements were extracted and TFs were predicted to complete the pathways. Comparing the similarities and differences is of significance in distinguishing key pathways and elements. The regulatory pathways of differentially expressed elements have an important effect on the progression of HL and abnormal modulation of the pathways results in the development of HL.

\section{Materials and methods}

Material collection and data processing. An experimentally validated dataset of human miRNAs and their targets was extracted from Tarbase 5.0 (http://diana.cslab.ece.ntua.gr/ tarbase/) and miRTarBase (http://mirtarbase.mbc.nctu.edu. tw/). The official and unified symbol of National Center for Biotechnology Information (NCBI) database was used to unify all miRNAs and genes. The NCBI database may be accessed online at http://www.ncbi.nlm.nih.gov/gene/. These experimental data support the current study. The complete data may be located in supplement file 1 and are designated as set $A_{1}$.

All data sets may be obtained by request from zmhmay@ sina.com

A human experimentally-validated dataset of TFs and miRNAs was extracted from TransmiR (15). Data from TransmiR was obtained from public literature and biological experiments. The complete data are designated as set $A_{2}$.

The host gene of human miRNA was manually extracted from miRBase (16) and NCBI. The official symbol and official ID was used to sign each host gene. The complete data are designated as set $A_{3}$.

In the current study, the differentially-expressed genes, including genetic mutation, abnormally expressed protein, SNPs, over expression genes, downregulated, upregulated and variation genes, were collected from Cancer Genetics Web (http://www.cancerindex.org/geneweb/index.html), NCBI SNP database (http://www.ncbi.nlm.nih.gov/snp/) and the relevant literature. HL-associated genes were collected from GeneCards database (17) and relevant literature, including genes that affect tumor growth, migration, radiation therapy, prevention, diagnosis, development and the clinical outcome of human HL (18). Differentially expressed elements were considered to be associated elements. In addition, 35 important TFs were extracted by the P-match method (19). These were considered to be HL-associated genes and the TFs included in transmiR were focused on. Promoter region sequences $(1,000 \mathrm{nt})$ of targets that are targeted by differentially-expressed miRNAs were downloaded from the UCSC database (20). The P-match method, which combines pattern matching and weight matrix approaches to identify transcription factor binding sites (TFBSs) in 1,000 nt promoter region sequences, was used and TFBSs were mapped onto the promoter region of targets. The matrix library of P-match, as well as sets of known TF-binding sites were collected in TRANSFAC to search for a large variety of different TF binding sites. The vertebrate matrix was used with restricted high quality criterion for the matrix. The complete data are designated as set $A_{4}$.

Differentially expressed miRNAs included overexpressed, downregulated and upregulated miRNAs. Differentially expressed miRNAs were collected from mir2Disease (21). Relevant literature was manually observed to extract HL-associated miRNAs. The complete data are designated as set $A_{5}$.

Three networks construction. The following method was used to construct the differentially expressed network, associated network and global network. All regulatory associations between TFs, miRNAs, targets and host genes were extracted from $A_{1}, A_{2}$ and $A_{3}$. Following the combination of all associations, the global network was constructed. Differentially expressed elements were separately extracted from $A_{4}$ and $A_{5}$ and the association between differentially expressed elements from the global network were selected, resulting in the differentially expressed network. A similar method was used to construct the related network. The complete data is located for differentially expressed network in supplement file 6 , for the related network in supplement file 7 and the global network in supplement file 8 .

\section{Results}

Differentially-expressed network of HL. Fig. 1 shows the significant regulatory associations between differentially expressed elements in HL. This network is composed of five TFs, TP53, MYC, NFKB1, JUN and REL, targets of miRNAs, 41 miRNAs and their host genes. The elements are all differentially-expressed except from the host genes. Fig. 1 shows three types of associations between each pair in HL, which are miRNA targets and host genes, including miRNAs and genes regulating miRNAs. A number of specific features of TFs and miRNAs are highlighted in Fig. 1. For example, hsa-miR-98 targets MYC, which regulates has-let-7a (7a-1, 7a-2 and 7a-3) and has-miR-9 (9-1, 9-2 and 9-3). Nie et al (22) hypothesized that has-let-7a and has-miR-9 target PRDM1 in HL cell lines, indicating that MYC indirectly affects 


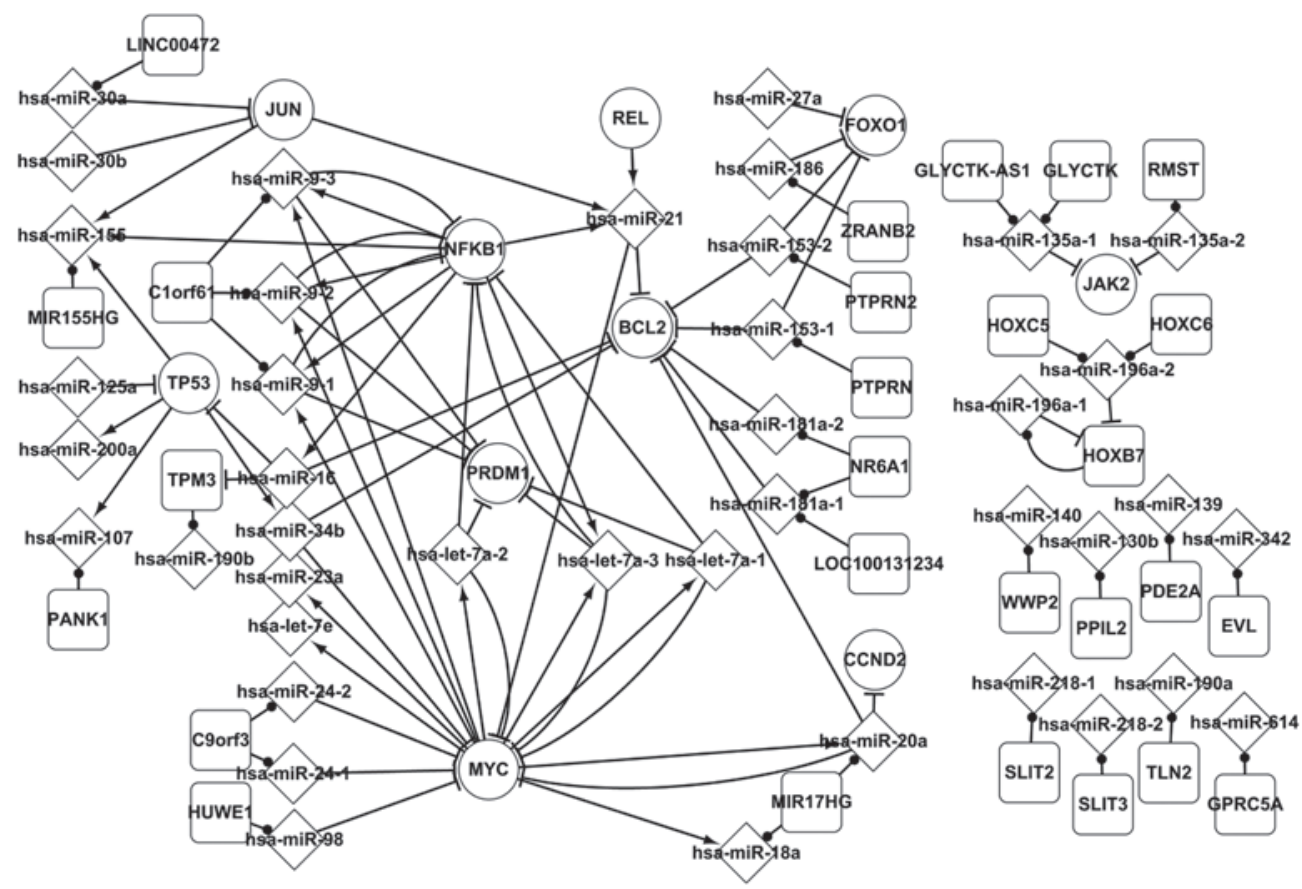

Figure 1. Differentially expressed networks of the genes and microRNAs in Hodgkin's lymphoma.

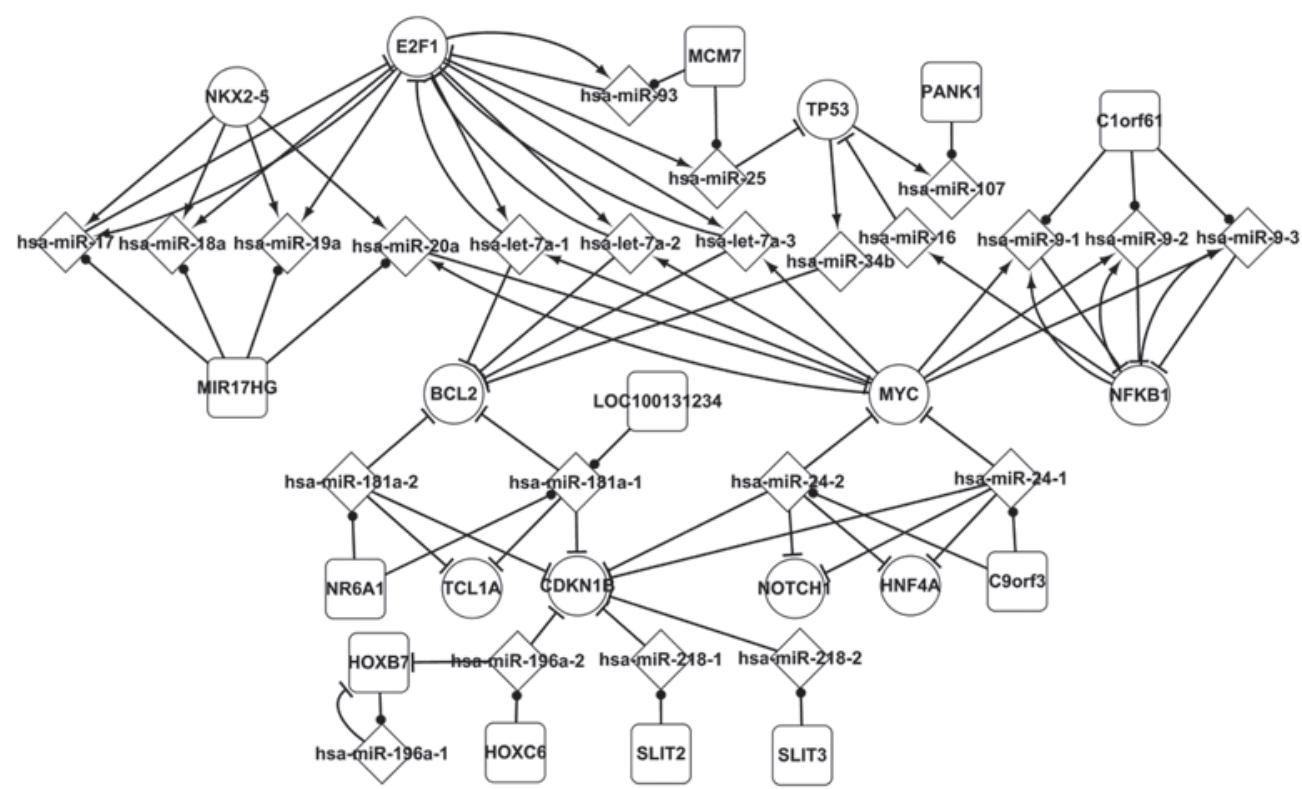

Figure 2. Host genes and the microRNAs of the differentially expressed networks in Hodgkin's lymphoma.

PRDM1 by has-let-7a and has-miR-9. A self-adaptation association was identified between MYC and has-let-7a, MYC and has-miR-20a and NFKB1 and has-miR-9. A number of specific features between miRNAs and their host genes are highlighted in Fig. 1. A host gene may include one or several miRNAs targeting other genes. For example, C9orf3 includes hsa-miR-24-1 and hsa-miR-24-2, which target MYC, CDKN1B, HNF4A and NOTCH1. An miRNA may be located in one or several genes. For example hsa-miR-181a-1 is located in NR6A1 and LOC100131234. The differentially expressed network partly revealed the regulatory mechanism of HL.
Related network of HL. The HL-associated network contains numerous regulatory correlations between genes and miRNAs. The associated network includes the differentially-expressed network. The associated network includes five differentially expressed TFs, 16 additional TFs, 57 miRNAs and a number of additional targets. The network shows mass additional pathways of genes and miRNAs. For instance, ZEB1 and GATA3 regulate hsa-miR-200a that, in turn, regulates ZEB1 and TFRC. SPI1 regulates hsa-miR-17, which targets BCL2. Gibcus et al (23) indicated that in HL, high has-miR-17 expression contributes to a dysfunctional p53 pathway and thereby to the malignant phenotype. Smolewski et al (24) showed that 


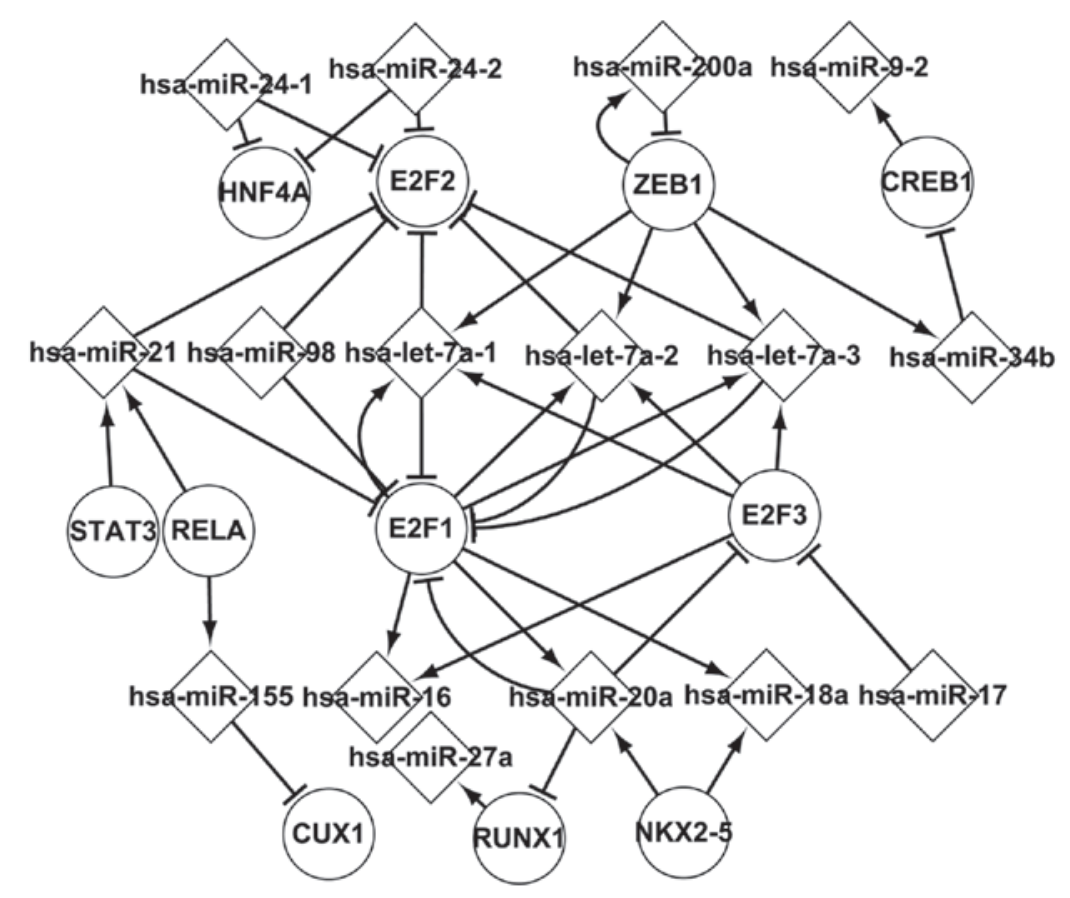

Figure 3. Transcription network of predicted transcription factors and differentially expressed microRNAs as well as target genes in Hodgkin's lymphoma.

BCL2 may be considered to be a novel prognostic factor in Hodgkin's disease. E2F1 regulates hsa-miR-25, which targets TP53 (25). Tanzer et al (26) demonstrated that hsa-miR-25 is involved in the pathophysiology of HL. Mutation of TP53 is involved in the pathogenesis of HL (27). The associated network expands additional topology associations of differentially expressed elements and contributes to the understanding of the progression of HL.

Global network of $H L$. The global network includes more comprehensive regulatory correlations that are from the three sets, $A_{1}, A_{2}$ and $A_{3}$ and is an experimentally validated biological network in the human body. The global network includes the differentially expressed and associated networks.

Host genes and the associated miRNAs in HL. In the current study, the host genes and their miRNAs showed important characteristics. Although host genes are not differentially expressed in HL, they were considered as differentially expressed genes if their miRNAs were differentially expressed. Numerous associations between host genes and other elements are shown in Fig. 2. MIR17HG includes four miRNAs, hsa-miR-17, hsa-miR-18a, hsa-miR-19a and hsa-miR-20a, which are regulated by NKX2-5 and E2F1. Hsa-miR-17 and hsa-miR-20a separately form a self-adaptation association with E2F1 and MYC. MCM7 includes two miRNAs, hsa-miR-93 and hsa-miR-25, which are regulated by E2F1. C1orf61 includes hsa-miR-9, which forms a self-adaptation association with NFKB1. NR6A1 includes hsa-miR-181a-1 and hsa-miR-181a-2, and combined they target BCL2, TCL1A and CDKN1B. Notably, HOXB7 includes hsa-miR-196a-1 and it is targeted by hsa-miR-196a-1. Host genes are important for determination of associations linking specific miRNAs and may aid in understanding the pathology of HL.
Transcriptional network of predicted TFs. Sixteen differentially expressed miRNAs, which are regulated by predicted TFs, were analyzed further. Fig. 3 shows the regulatory associations of predicted TFs and differentially-expressed miRNAs in HL. These elements sequentially affect their successors by targeting or regulating them. Fig. 3 shows that ZEB1, E2F1 and E2F3 coregulate has-let-7a, which targets E2F2. From Fig. 3 it is possible to conclude that a differentially expressed miRNA may target several TFs, a TF may regulate several differentially expressed miRNAs, a TF indirectly affects other TFs by several differentially expressed miRNAs and a differentially expressed miRNA indirectly affects other miRNAs by TF. For example, hsa-miR-20a targets E2F1, E2F3 and RUNX1; ZEB1 regulates hsa-miR-200a, hsa-miR-34b and hsa-miR-7a; NKX2-5 indirectly regulates RUNX1 by hsa-miR-20a; and hsa-miR-17 indirectly affects hsa-miR-16 by E2F3. The transcription network of predicted TFs and miRNAs is important for the analysis of the pathogenesis of HL.

Regulatory associations of differentially-expressed genes. To describe the network of HL more clearly, the upstream and downstream information of differentially expressed elements, genes and miRNAs, were extracted and compared, and the TFs were predicted from the P-match method. The successor nodes and precursor nodes of the differentially expressed genes from the three networks were extracted, classed and listed. Specific regulatory associations were highlighted from the list. Among these genes, MYC and NFאB1 revealed an important characteristic involving specific common precursor and on elements in the successor nodes, indicating that the gene and miRNAs form a self-adaptation association. The complete data may be identified in supplement file 9.

TFs were selected, the first class of which, had six types of adjacent nodes (three types of successors and three types of 
Table I. Regulatory associations between miRNAs and MYC in Hodgkin's lymphoma.

A. miRNAs that target MYC in the three networks.

\begin{tabular}{lll}
\hline $\begin{array}{l}\text { Differentially } \\
\text { expressed } \\
\text { network }\end{array}$ & $\begin{array}{c}\text { Associated } \\
\text { network }\end{array}$ & $\begin{array}{c}\text { Global } \\
\text { network }\end{array}$ \\
\hline $\begin{array}{ll}\text { let-7a-1 } \\
\text { let-7a-2 }\end{array}$ & $\begin{array}{l}\text { let-7a-1 } \\
\text { let-7a-2 }\end{array}$ & $\begin{array}{l}\text { let-7a-1 } \\
\text { let-7a-2 }\end{array}$ let-7a-3 \\
miR-20a & miR-20a & let-7a-3 \\
miR-21 & miR-21 & miR-20a \\
miR-24-1 & miR-24-1 & miR-24-1 \\
miR-24-2 & miR-24-2 & miR-24-2 \\
miR-34b & miR-34b & miR-34b \\
miR-98 & miR-98 & miR-98 \\
& miR-17 & miR-17 \\
& & let-7g \\
& & miR-145 \\
& & miR-26a \\
& & miR-34a \\
& & miR-30c-5p \\
& & miR-378 \\
\hline
\end{tabular}

B. miRNAs regulated by MYC in the three networks.

\begin{tabular}{|c|c|c|}
\hline $\begin{array}{l}\text { Differentially } \\
\text { expressed } \\
\text { network }\end{array}$ & $\begin{array}{l}\text { Associated } \\
\text { network }\end{array}$ & $\begin{array}{c}\text { Global } \\
\text { network }\end{array}$ \\
\hline $\begin{array}{l}\text { let-7a-1 } \\
\text { let-7a-2 } \\
\text { let-7a-3 } \\
\text { miR-20a } \\
\text { miR-18a } \\
\text { miR-23a } \\
\text { miR-9-1 } \\
\text { miR-9-2 } \\
\text { miR-9-3 }\end{array}$ & $\begin{array}{l}\text { let-7a-1 } \\
\text { let-7a-2 } \\
\text { let-7a-3 } \\
\text { miR-20a } \\
\text { miR-18a } \\
\text { miR-23a } \\
\text { miR-9-1 } \\
\text { miR-9-2 } \\
\text { miR-9-3 } \\
\text { miR-17 } \\
\text { miR-19a } \\
\text { miR-19b } \\
\text { miR-106a } \\
\text { miR-106a } \\
\text { miR-106a } \\
\text { miR-93 }\end{array}$ & $\begin{array}{l}\text { let-7a-1 } \\
\text { let-7a-2 } \\
\text { let-7a-3 } \\
\text { miR-20a } \\
\text { miR-18a } \\
\text { miR-23a } \\
\text { miR-9-1 } \\
\text { miR-9-2 } \\
\text { miR-9-3 } \\
\text { miR-17 } \\
\text { miR-19a } \\
\text { miR-19b } \\
\text { miR-106a } \\
\text { miR-106a } \\
\text { miR-106a } \\
\text { miR-93 } \\
\text { miR-16-1 } \\
\text { miR-34a } \\
\text { miR-23b } \\
\text { miR-141 } \\
\text { miR-221 } \\
\text { miR-195 } \\
\text { miR-22 } \\
\text { miR-29a } \\
\text { miR-29c } \\
\text { miR-26b } \\
\text { miR-26a } \\
-7 b, 1 e t-7 c\end{array}$ \\
\hline
\end{tabular}

Table I. Continued.

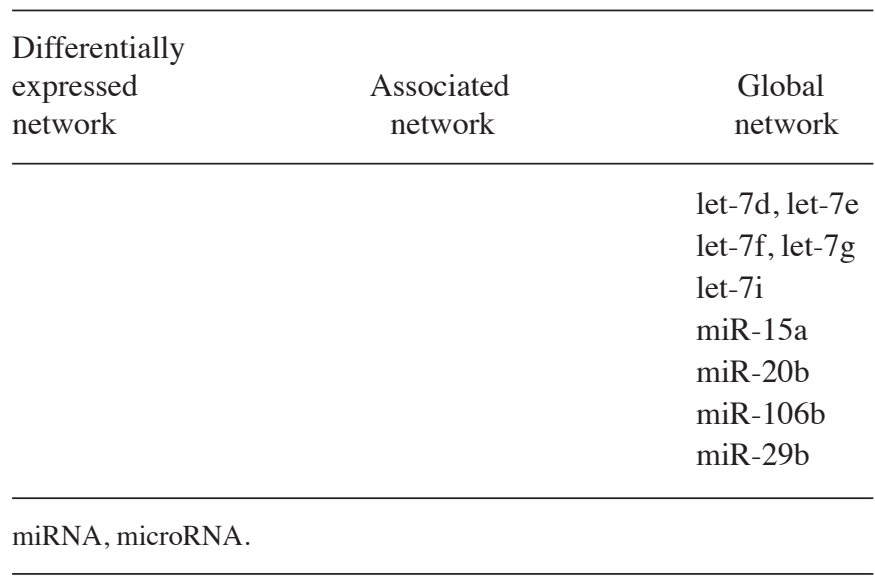

predecessors). This type of TF includes JUN, MYC, NFкB1 and TP53. The subsequent experiments focused on MYC.

In Table I, the precursors and successors of MYC in the differentially-expressed, associated and global networks are listed. MYC is a notable tumor suppressor and has significant features in the three networks. Table I shows that nine miRNAs target MYC and MYC regulates nine miRNAs in the differentially expressed network. In the present study it has been hypothesized that the precursors of MYC indirectly affect the successors expression via MYC. Hsa-miR-20a and hsa-let-7a target MYC and MYC regulates them in return, forming a self-adaptation correlation. Supplement file 9 shows that MYC indirectly affects other genes via a number of miRNAs. For example, MYC regulates hsa-miR-17, which targets BCL2. Numerous TFs also indirectly affect MYC via specific miRNAs. For example, TP53 and NFкB1 regulate hsa-miR-34, which targets MYC.

The second class of TF had three types of adjacency nodes (three types of successors), including REL. There is no miRNA targeting REL and it regulates hsa-miR-21 in three networks.

In addition, the remaining genes that were not TFs were investigated. The first class of genes had three types of adjacency nodes, (three types of predecessors), including BCL2, CCND2, FGFR3, FOXO1, JAK2 and PRDM1. The adjacency nodes were identified to be targeted by specific miRNAs and did not regulate any miRNA.

The second class of gene, CD44, had a type of adjacency node in three networks, which is a type of predecessor. It is hypothesized that CD44 has the least effect compared with other differentially expressed genes.

Regulatory associations of differentially expressed miRNAs. The regulatory associations of each differentially expressed miRNA were extracted, compared and analyzed by the same method as differentially expressed genes. The complete data may be located in supplement file 10. Among these miRNAs, seven differentially expressed miRNAs, hsa-let-7a-1, hsa-let-7a-2, hsa-let-7a-3, hsa-miR-20a, hsa-miR-9-1, hsa-miR-9-2 and hsa-miR-9-3 and corresponding genes, form self-adaptation.

The first class of miRNA had six types of adjacent nodes, three types of predecessors and three types of successors, 
Table II. Regulatory relations between hsa-miR-20a and genes in Hodgkin's lymphoma.

A. Genes that regulate hsa-miR-20a in the three networks.

\begin{tabular}{lll}
\hline $\begin{array}{l}\text { Differentially } \\
\text { expressed } \\
\text { network }\end{array}$ & $\begin{array}{l}\text { Related } \\
\text { network }\end{array}$ & $\begin{array}{l}\text { Global } \\
\text { network }\end{array}$ \\
\hline MYC & MYC & MYC \\
& NKX2-5 & NKX2-5 \\
CCND1 & CCND1 \\
& E2F1 & E2F1 \\
& SPI1 & SPI1 \\
& & TLX1 \\
& & TLX3 \\
& & ERS1 \\
& & STAT5 \\
\hline
\end{tabular}

B. Genes targeted by the hsa-miR-20a in the three networks.

\begin{tabular}{lll}
\hline $\begin{array}{l}\text { Differentially } \\
\text { expressed } \\
\text { network }\end{array}$ & $\begin{array}{l}\text { Related } \\
\text { network }\end{array}$ & \multicolumn{1}{c}{$\begin{array}{c}\text { Global } \\
\text { network }\end{array}$} \\
\hline MYC & MYC & MYC, MAP3K12 \\
BCL2 & BCL2 & BCL2, WEE1 \\
CCND2 & CCND2 & CCND2, RB1 \\
& E2F1 & E2F1, CDKN1A \\
E2F3 & E2F3, HIF1A \\
& RBL1 & RBL1, RBL2 \\
& CCND1 & CCND1, APP \\
& RUNX1 & RUNX1,NRAS \\
& & SMAD4, PTEN \\
& & MUC17, BMPR2 \\
& & VEGFA, BNIP2 \\
& & MAPK9,TGFBR2 \\
& & MEF2D, THBS1 \\
\hline
\end{tabular}

including hsa-miR-20a and hsa-miR-34b. The subsequent experiments focused on hsa-miR-20a.

In Table II, the precursors and successors of hsa-miR-20a in the differentially expressed, associated and global networks are listed. Table II shows that MYC regulates hsa-miR-20a, as well as targeting three genes in the differentially expressed network. MYC has been concluded to indirectly affect MYC, BCL2 and CCND2 expression through hsa-miR-20a. Table II shows that MYC and hsa-miR-20a, CCND1 and hsa-miR-20a and E2F1 and hsa-miR-20a separately form self-adaptation. Supplement file 10 shows that hsa-miR-20a also indirectly affects other miRNAs by specific TFs, for example hsa-miR-20a targets E2F1 that regulates hsa-let-7a, hsa-miR-16 and hsa-miR-18a. A number of miRNAs also indirectly affect hsa-miR-20a by targeting certain TFs, for example hsa-miR-155 targets SPI, which regulates hsa-miR-20a.

The second class of miRNA had five types of adjacency nodes (two types of successors and three types of predecessors, three types of successors and two types of predecessors), including hsa-miR-155 and hsa-miR-27a.
Table III. Regulatory relations between miRNAs and E2F1 in Hodgkin's lymphoma.

A. miRNAs that target E2F1 in the three networks.

\begin{tabular}{lll}
\hline $\begin{array}{l}\text { Differentially } \\
\text { expressed } \\
\text { network }\end{array}$ & $\begin{array}{l}\text { Related } \\
\text { network }\end{array}$ & $\begin{array}{l}\text { Global } \\
\text { network }\end{array}$ \\
\hline let-7a-1 & let-7a-1 & let-7a-1 \\
let-7a-2 & let-7a-2 & let-7a-2 \\
let-7a-3 & let-7a-3 & let-7a-3 \\
miR-20a & miR-20a & miR-20a \\
miR-21 & miR-21 & miR-21 \\
miR-98 & miR-98 & miR-98 \\
& miR-106a & miR-106a \\
& miR-17 & miR-17 \\
& miR-93 & miR-93 \\
& & miR-34a \\
& & miR-106b \\
& & miR-126 \\
& & miR-223 \\
& & miR-330 \\
& & miR-149 \\
\hline
\end{tabular}

B. miRNAs regulated by $\mathrm{E} 2 \mathrm{~F} 1$ in the three networks.

\begin{tabular}{|c|c|c|}
\hline $\begin{array}{l}\text { Differentially } \\
\text { expressed } \\
\text { network }\end{array}$ & $\begin{array}{l}\text { Related } \\
\text { network }\end{array}$ & $\begin{array}{c}\text { Global } \\
\text { network }\end{array}$ \\
\hline $\begin{array}{l}\text { let-7a-1 } \\
\text { let-7a-2 } \\
\text { let-7a-3 } \\
\text { miR-16 } \\
\text { miR-18a } \\
\text { miR-20a }\end{array}$ & $\begin{array}{l}\text { let-7a-1 } \\
\text { let-7a-2 } \\
\text { let-7a-3 } \\
\text { miR-16 } \\
\text { miR-18a } \\
\text { miR-20a } \\
\text { miR-106a } \\
\text { miR-17 } \\
\text { miR-19a } \\
\text { miR-19b } \\
\text { miR-25 } \\
\text { miR-92a } \\
\text { miR-93 }\end{array}$ & $\begin{array}{l}\text { let-7a-1 } \\
\text { let-7a-2 } \\
\text { let-7a-3 } \\
\text { miR-16 } \\
\text { miR-18a } \\
\text { miR-20a } \\
\text { miR-106a } \\
\text { miR-17 } \\
\text { miR-19a } \\
\text { miR-19b } \\
\text { miR-25 } \\
\text { miR-92a } \\
\text { miR-93 } \\
\text { miR-15a } \\
\text { miR-15b } \\
\text { miR-195 } \\
\text { miR-106b } \\
\text { let-7i } \\
\text { miR-18b } \\
\text { miR-20b } \\
\text { miR-223 } \\
\text { miR-363 } \\
\text { miR-449a } \\
\text { miR-449b } \\
\text { miR-449c }\end{array}$ \\
\hline
\end{tabular}

miRNA, microRNA. 
The third class of miRNA had four types of adjacency nodes, (three types of successors and a type of predecessor, two types of successors and two types of predecessors and a type of successor and three types of predecessors), including hsa-miR-125a and hsa-let-7e.

The fourth class of miRNA had three types of adjacency nodes (three types of successors, two types of successors and a kind of predecessor and a type of successor and two types of predecessors), including hsa-miR-98, hsa-miR-130b and hsa-miR-206.

The fifth class of miRNA had two types of adjacency nodes (two types of successors or a type of successor and a type of predecessor), including hsa-miR-150 and hsa-miR-140.

The sixth class of miRNA had a type of adjacency node (a type of successor), including hsa-miR-196a-1.

Regulatory associations of predicted transcription factors. Using the aforementioned method, predicted TF data from the P-match method was processed. The complete data may be located in supplement file 10. Two TFs, E2F1 and ZEB1, and corresponding miRNAs were identified to form self-adaptation associations.

The first class of TF had six types of adjacent nodes (three types of successors and three types of predecessors), including CREB1, E2F1, E2F3, RUNX1 and ZEB1. The subsequent experiments focused on E2F1.

In Table III, the precursors and successors of E2F1 in the differentially expressed, associated and global networks are listed. There were six differentially expressed miRNAs that target E2F1 and E2F1 regulates six differentially expressed miRNAs. Four of which, hsa-let-7a-1, hsa-let-7a-2, hsa-let-7a-3 and hsa-miR-20a, separately form self-adaptation with E2F1 in differentially expressed networks.

The second class of TF had four types of adjacent nodes (three types of successors and a type of predecessors), including STAT3 and RELA.

The third class of TF had three types of adjacent nodes (three types of successors or three types of predecessors), including NKX2-5 and HNF4A.

The fourth class of TF had two types of adjacent nodes (two types of successors or a type of successors and a type of predecessor), including HOXA5 and YY1.

The fifth class of TF has one adjacent node (a type of successor or a type of predecessor), including TCF3 and PLAU.

\section{Discussion}

The present study highlights a number of important pathways of differentially-expressed genes, differentially expressed miRNAs and predicted TFs in HL.

For the differentially-expressed elements, the current results show numerous important pathways, including three or more elements. For example, hsa-miR-30a targets JUN and JUN regulates hsa-miR-21. These pathways exhibit a key biological function in HL. Certain pathways have not only been observed in HL, but also in other carcinomas. For example, hsa-miR-16 induces apoptosis by targeting BCL2 in chronic lymphocytic leukemia (28). Hsa-miR-16 was hypothesized to target the BCL2 pathway, thus, the role of hsa-miR-16 requires further investigation in studies of carcinoma. Pathways in HL have been observed to be associated with the progression of various other carcinomas. For example, hsa-miR-19a targets BCL2L11 in T-cell acute lymphoblastic leukemia (29).

The TFs predicted from the P-match method indicate the potential associations between the differentially expressed miRNAs and TFs. Further studies are required to validate the close associations between HL and miRNAs and TFs. For the pathways of predicted TFs, a number have been identified in other carcinomas, for example, NKX2-5 targets miR-17-92 and concomitantly reduces E2F1, thereby enhancing the survival rate of leukemic T-cells (30). This is likely to enable further knowledge of the linkage between genes in various carcinomas and highlight current gaps in research in the pathology of HL.

In the present study, three regulatory topological networks of elements involved in HL were constructed. Three figures were extracted from the networks to highlight certain important pathways and elements. To further investigate the network, the successors and precursors of the elements in the three networks were investigated. The entire experimentally validated data of HL are presented in the current study. A number of were formed with the P-match method, which provides specific starting points for further studies of HL. Future studies are likely to focus on the interaction between proteins and the regulatory patterns (upregulation and downregulation) enabling the construction of a comprehensive and extensive network of HL.

\section{Acknowledgements}

This study was supported by grants from the National Natural Science Foundation of China (grant nos. 60973091 and 60905022).

\section{References}

1. Gibcus JH, Tan LP, Harms G, et al: Hodgkin lymphoma cell lines are characterized by a specific miRNA expression profile. Neoplasia 11: 167-176, 2009.

2. Xie L, Ushmorov A, Leithäuser F, et al: FOXO1 is a tumor suppressor in classical Hodgkin lymphoma. Blood 119: 3503-3511, 2012.

3. Hobert O: Gene regulation by transcription factors and microRNAs. Science 319: 1785-1786, 2008.

4. Tran DH, Satou K, Ho TB and Pham TH: Computational discovery of miR-TF regulatory modules in human genome. Bioinformation 4: 371-377, 2010.

5. Li M, Li J, Ding X, He M and Cheng SY: microRNA and cancer. AAPS J 12: 309-317, 2010.

6. Naeem H, Küffner R and Zimmer R: MIRTFnet: analysis of miRNA regulated transcription factors. PLoS One 6: e22519, 2011.

7. Xiao F, Zuo Z, Cai G, et al: miRecords: an integrated resource for microRNA-target interactions. Nucleic Acids Res 37 (Database issue): D105-D110, 2009.

8. Betel D, Wilson M, Gabow A, Marks DS and Sander C: The microRNA.org resource: targets and expression. Nucleic Acids Res 36: D149-D153, 2008.

9. Papadopoulos GL, Reczko M, Simossis VA, Sethupathy P and Hatzigeorgiou AG: The database of experimentally supported targets: a functional update of TarBase. Nucleic Acids Res 37: D155-D158, 2009.

10. Hsu SD, Lin FM, Wu WY, et al: miRTarBase: a database curates experimentally validated microRNA-target interactions. Nucleic Acids Res 39: D163-D169, 2011.

11. Rodriguez A, Griffiths-Jones S, Ashurst JL and Bradley A: Identification of mammalian microRNA host genes and transcription units. Genome Res 14: 1902-1910, 2004. 
12. Baskerville S and Bartel DP: Microarray profiling of microRNAs reveals frequent coexpression with neighboring miRNAs and host genes. RNA 11: 241-247, 2005.

13. Cao G, Huang B, Liu Z, et al: Intronic miR-301 feedback regulates its host gene, ska2, in A549 cells by targeting MEOX2 to affect ERK/CREB pathways. Biochem Biophys Res Commun 396: 978-982, 2010 .

14. Gibcus JH, Tan LP, Harms G, et al: Hodgkin lymphoma cell lines are characterized by a specific miRNA expression profile. Neoplasia 11: 167-176, 2009.

15. Wang J, Lu M, Qiu C and Cui Q: TransmiR: a transcription factor-microRNA regulation database. Nucleic Acids Res 38: D119-D122, 2010.

16. Kozomara A and Griffiths-Jones S: miRBase: integrating microRNA annotation and deep-sequencing data. Nucleic Acids Res 39: D152-D157, 2011.

17. Safran M, Dalah I, Alexander J, et al: GeneCards Version 3: the human gene integrator. Database (Oxford) 2010: baq020, 2010.

18. Huang da W, Sherman BT, Lempicki RA: Systematic and integrative analysis of large gene lists using DAVID bioinformatics resources. Nature Protoc 4: 44-57, 2009.

19. Chekmenev DS, Haid C and Kel AE: P-Match: transcription factor binding site search by combining patterns and weight matrices. Nucleic Acids Res 33: W432-W437, 2005.

20. Fujita PA, Rhead B, Zweig AS, et al: The UCSC Genome Browser database: update 2011. Nucleic Acids Res 39: D876-D882, 2011.

21. Jiang Q, Wang Y, Hao Y, et al: miR2Disease: a manually curated database for microRNA deregulation in human disease. Nucleic Acids Res 37: D98-D104, 2009.

22. Nie K, Gomez M, Landgraf P, et al: MicroRNA-mediated down-regulation of PRDM1/Blimp-1 in Hodgkin/Reed-Sternberg cells: a potential pathogenetic lesion in Hodgkin lymphomas. Am J Pathol 173: 242-252, 2008.
23. Gibcus JH, Kroesen BJ, Koster R, et al: MiR-17/106b seed family regulates p21 in Hodgkin's lymphoma. J Pathol 225: 609-617, 2011.

24. Smolewski P, Niewiadomska H, Błonski JZ, Robak T and Krykowski E: Expression of proliferating cell nuclear antigen (PCNA) and p53, bcl-2 or C-erb B-2 proteins on Reed-Sternberg cells: prognostic significance in Hodgkin's disease. Neoplasma 45: 140-147, 1998.

25. Massip A, Arcondéguy T, Touriol C, et al: E2F1 activates p53 transcription through its distal site and participates in apoptosis induction in HPV-positive cells.

26. FEBS Lett 587: 3188-3194, 2013

27. Tanzer A and Stadler PF: Molecular evolution of a microRNA cluster. J Mol Biol 339: 327-335, 2004.

28. Feuerborn A, Möritz C, Von Bonin F, Dobbelstein M, Trümper L, Stürzenhofecker B and Kube D: Dysfunctional p53 deletion mutants in cell lines derived from Hodgkin's lymphoma. Leuk Lymphoma 47: 1932-1940, 2006.

29. Cimmino A, Calin GA, Fabbri M, et al: miR-15 and miR-16 induce apoptosis by targeting BCL2. Proc Natl Acad Sci USA 102: 13944-13949, 2005.

30. Ye H, Liu X, Lv M, et al: MicroRNA and transcription factor co-regulatory network analysis reveals miR-19 inhibits CYLD in T-cell acute lymphoblastic leukemia. Nucleic Acids Res 40: 5201-5214, 2012.

31. Nagel S, Venturini L, Przybylski GK, et al: Activation of miR-17-92 by NK-like homeodomain proteins suppresses apoptosis via reduction of E2F1 in T-cell acute lymphoblastic leukemia. Leuk Lymphoma 50: 101-108, 2009. 